\title{
La espondilitis anquilosante, causas y diagnóstico. Una revisión sistemática
}

Ankylosing spondylitis, causes and diagnosis. A systematic review

1 Geovanny German Cañar Lascano

https://orcid.org/0000-0003-3424-3740

Universidad Católica de Santiago de Guayaquil, Guayaquil, Ecuador geovanny.canar@cu.ucsg.edu.ec

2 Alberto Sper Sempértegui

https://orcid.org/ 0000-0003-4793-9704

Universidad Católica de Santiago de Guayaquil, Guayaquil, Ecuador

alberto.sper@cu.ucsg.edu.ec

3 Juan Gonzalo Fernández Villarroel Investigador Independiente, Ecuador

(iD https://orcid.org/0000-0002-2037-5657 jfshapes@hotmail.co.uk

Artículo de Investigación Científica y Tecnológica Enviado: 13/12/2021

Revisado: 29/12/2021

Aceptado: 28/01/2022

Publicado:05/02/2022

DOI: https://doi.org/10.33262/ap.v4i1.1.170

Chenche Jácome, W. L., \& Posligua Espinoza, J. E. (2022). Utilización de la TIC en los métodos tecno pedagógicos que inciden en la gestión docente . AlfaPublicaciones, 4(1.1), 448-472. https://doi.org/10.33262/ap.v4i1.1.170

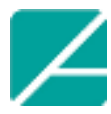

Ciencia

Digital

ALFA PUBLICACIONES, es una Revista Multidisciplinar, Trimestral, que se publicará en soporte electrónico tiene como misión contribuir a la formación de profesionales competentes con visión humanística y crítica que sean capaces de exponer sus resultados investigativos y científicos en la misma medida que se promueva mediante su intervención cambios positivos en la sociedad. https://alfapublicaciones.com

La revista es editada por la Editorial Ciencia Digital (Editorial de prestigio registrada en la Cámara Ecuatoriana de Libro con No de Afiliación 663) www.celibro.org.ec

Esta revista está protegida bajo una licencia Creative Commons Attribution Non Commercial No Derivatives

4.0 International. Copia de la licencia: http://creativecommons.org/licenses/by-nc-nd/4.0/ 


\section{Palabras}

\section{claves:}

Espondilitis anquilosante, calidad de vida, genética, dolor, inflamación.

\section{Keywords:}

Ankylosing spondylitis, quality of life, genetics, pain, inflammation.

\section{Resumen}

La espondilitis anquilosante es una artritis que se caracteriza por presentar inflamación crónica y las articulaciones se van degenerando, que afecta en gran proporción la columna vertebral y la articulación sacro ilíaca, esta enfermedad es más común que se presente en hombre que en mujeres, alrededor de los 20 a 30 años, afectado la calidad de vida de los individuos, y generando malestar y limitación de su movilidad. El objetivo de esta investigación es describir las causas y el diagnóstico de la espondilitis anquilosante a través de una revisión sistemática. Como conclusión se menciona que, es evidente que existe una gran variedad de afecciones que pueden estar asociadas con la EA, sin embargo, se observa la coincidencia que una de las principales consecuencias que genera esta enfermedad es el deterioro de la calidad de vida de los individuos que padecen esta y otras enfermedades durante su vida, y que repercuten en el comportamiento psicológico de los mismos. Es importante destacar que se debe seguir realizando análisis y estudios genéticos y de casos de pacientes con esta patología que permita determinar de forma más clara las causas que generan esta enfermedad y la prevalencia de la misma en los individuos, así como poder determinar posibles nuevos tratamientos que permitan mejorar en gran medida los síntomas, como el dolor, la inflamación y la rigidez para que las personas con esta enfermedad puedan realizar sus actividades sin depender de la ayuda de otras personas.

\section{Abstract}

Ankylosing spondylitis is an arthritis that is characterized by chronic inflammation and the joints degenerate, which affects a large proportion of the spine and the sacroiliac joint, this disease is more common in men than in women, around the 20 to 30 years, affected the quality of life of individuals, and generating discomfort and limitation of their mobility. The objective of this research is to describe the causes and diagnosis of ankylosing spondylitis through a systematic review. In conclusion, it is mentioned that it is evident that there is a great variety of conditions that may be associated with $\mathrm{AD}$, however, the coincidence is observed that one of the main consequences generated by this disease is the deterioration of the quality of life of individuals. who suffer from this and other illnesses during their lives, and which have repercussions on their psychological behavior? It is important to note that genetic analyzes 
and studies and case studies of patients with this pathology should continue to be carried out in order to more clearly determine the causes that generate this disease and its prevalence in individuals, as well as to be able to determine possible new treatments. that greatly improve symptoms such as pain, swelling and stiffness so that people with this disease can carry out their activities without relying on the help of others.

\section{Introducción}

La anatomía humana es compleja, y está conformada por células, músculos, nervios y huesos que le dan forma a cada una de las partes del organismo. Uno de los elementos que conforma el cuerpo humano es la columna vertebral, la cual es una estructura igualmente compleja, que está ubicada en la parte medio y posterior del tronco que va desde el hueso occipital y termina en la pelvis (Rodríguez \& Pérez, 2021).

De igual manera, la estructura de la columna es osteofibrocartilaginosa y sus funciones principales son sostener y soportar la cabeza, proteger el cordón medular, y las raíces nerviosas que de ella emergen, resguarda órganos como el corazón y los pulmones, es el centro de gravedad del cuerpo humano pues permite la movilidad y flexibilidad del cuerpo, otras funciones biomecánicas que se mencionan es que representa el al poder soportar la mitad superior del cuerpo representa un $60 \%$ del peso total del individuo (Sierra et al., 2018; Sinchiguano, 2018).

Esta estructura en los hombres posee una longitud de $70 \mathrm{~cm}$ y en las mujeres de $60 \mathrm{~cm}$, es resistente y flexible y está conformada por vertebras, dispuestas una sobre otras que permiten sostener la cabeza, la caja torácica, los miembros superiores manteniendo el equilibrio y transmite el peso a los miembros inferiores (Chiriboga, 2010).

Asimismo, Sierra et al. (2018), menciona que la columna vertebral presenta dos pares de curvaturas fisiológicas que se muestran en la figura 1: 


\section{Figura 1}

Curvaturas de la Columna Vertebral

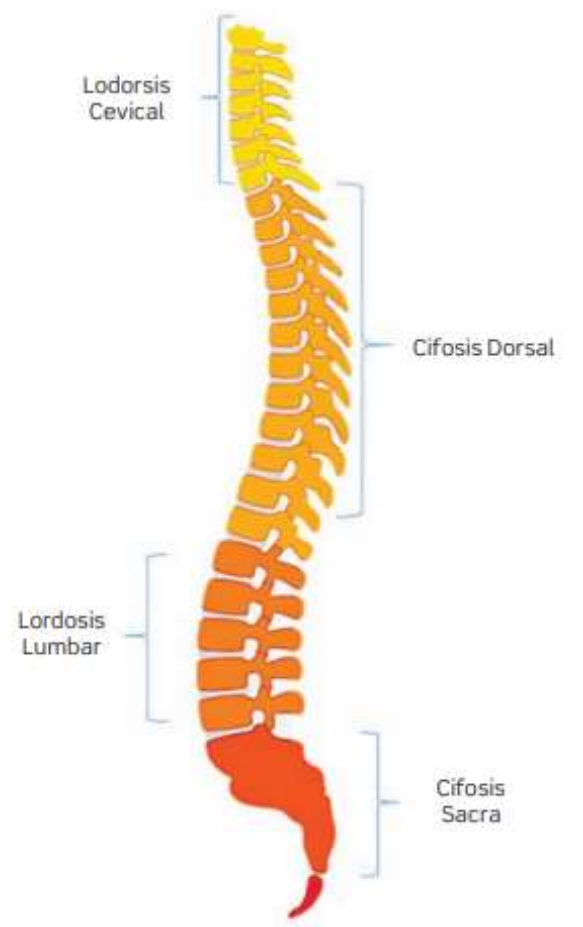

Fuente: Sierra et al. (2018)

En referencia a la figura anterior se menciona que estas curvaturas propias de la columna son de gran importancia y permiten relacionarlas con diversas patologías que puede presentar el ser humano, y que de acuerdo a las curvaturas se puede distinguir la edad, la masa corporal y hasta el género del individuo.

La columna vertebral posee entre 33 a 34 vertebras (ver figura 2) y se dividen de la siguiente manera:

- 7 Cervicales (C1-C7)

- 12 Torácicas (T1-T7)

- 5 Lumbares (L1-L5)

- 5 Sacras (S1-S5)

- 3 a 5 Coccígeas (Sinchiguano, 2018) 


\section{Figura 2}

Clasificación de las vertebras según su ubicación

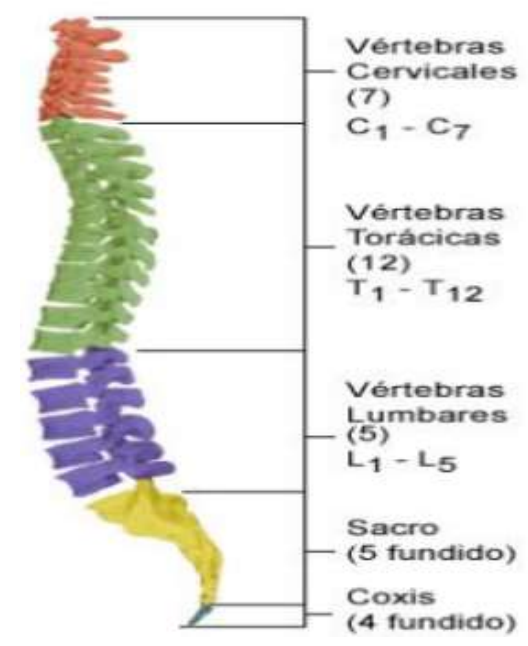

Fuente: Espinoza (2017)

De esta manera Chiriboga (2010), menciona que:

Cada vertebra está conformada por un segmento abultado que se le denomina cuerpo vertebral un arco óseo de concavidad anterior conocido como arco vertebral. Éstos rodean un espacio llamado agujero vertebral, este arco vertebral está formado a cada lado por dos pedículos en la porción anterior y por dos láminas en la posterior. Este arco óseo posee un saliente medio posterior, la apófisis espinosa, dos apófisis transversas y cuatro apófisis articulares. (p. 26)

En este sentido cada zona de la columna cumple una función específica, en el caso de la columna cervical por ejemplo, es la que posee mayor movilidad y permite que la cabeza se oriente en distintas direcciones, su curvatura es la menos pronunciada y convexa hacia adelante, la $\mathrm{C} 1$ denominada atlas es más larga que el resto de las cervicales no posee cuerpo ni apófisis espinosa, por arriba se une con los cóndilos del hueso occipital del cráneo y por debajo se une con la siguiente vertebra (axis) (C2) (ver figura 3) (Sinchiguano, 2018). 


\section{Figura 3}

Vertebras atlas y axis
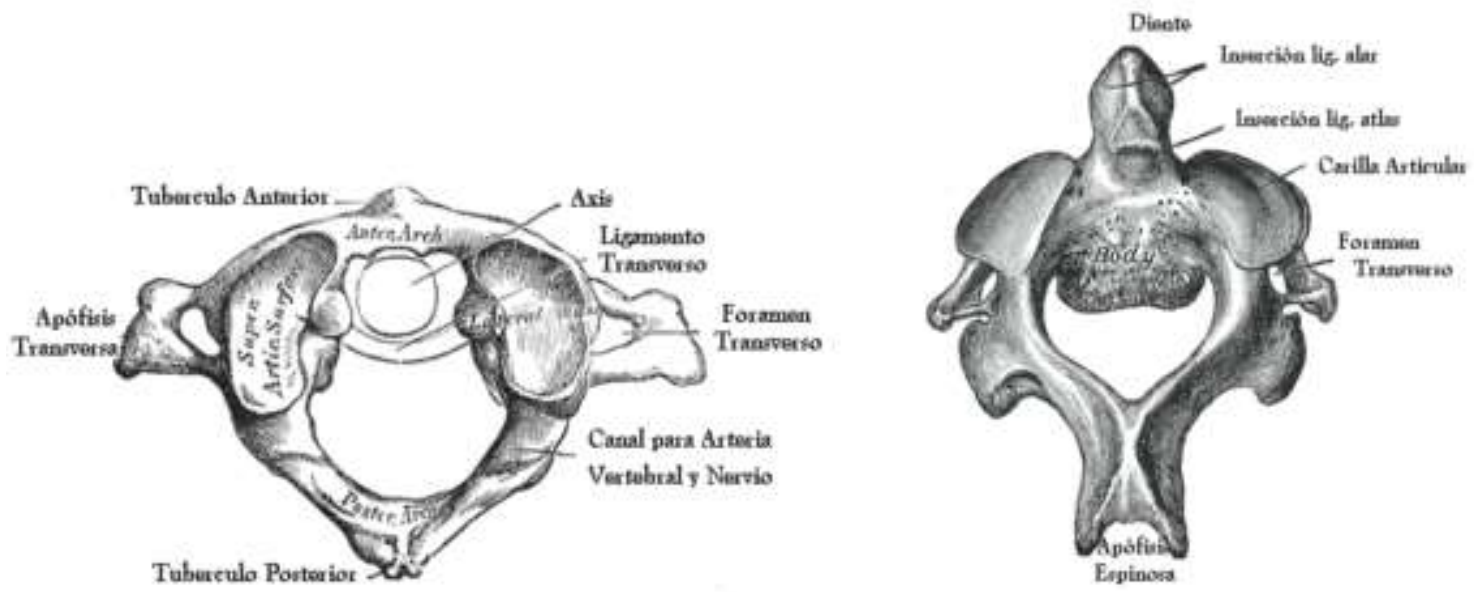

Fuente: Chiriboga (2010)

La vertebra C7 es la que se le denomina vertebra de transición Sinchiguano (2018), que posee una apófisis espinosa de gran longitud y una transversal, y que en conjunto con todas las demás vértebras cervicales realizan movimientos de extensión, flexión, rotación y latero flexión.

En la zona de la columna dorsal o torácica sus vertebras son más gruesas que las cervicales estas tienen forma de corazón, las apófisis espinosas son inclinadas hacia abajo, y el agujero vertebral es pequeño y con forma circular y están conformadas por facetas costales en las apófisis transversales que se unen de forma articulada en ambos lados con los tubérculos de las costillas (Chiriboga, 2010).

De firma más precisa se detallan sus características descritas por Chiriboga (2010) así:

- Los cuerpos son grandes y tienen forma de riñón

- La concavidad mira hacia el agujero vertebral triangular

- Los pedículos son cortos, gruesos y rígidos hacia atrás.

- Las láminas son cortas, gruesas y relativamente desiguales, extendiéndose por debajo del nivel de los pedículos.

- Las apófisis transversas son largas y delgadas. (p.30)

En cuanto a la vértebras lumbares Sinchiguano (2018), menciona que están conformadas por 5 vertebras desde la L1 a la L5 y están diseñadas para poder resistir la mayor parte del peso del cuerpo del individuo y se caracterizan por el volumen de la apófisis transversales y espinosas así como por la masa con la que están conformadas (ver Figura 4), además permite una movilidad de flexión $\left(60^{\circ}\right)$, extensión $\left(35^{\circ}\right)$, latero flexión $\left(20^{\circ}\right)$, rotación $\left(5^{\circ}\right)$, asimismo el agujero vertebral es triangular en estas vertebras, tanto la L1 
como la L5 poseen una estructura especial por ser las que se unen con la zona vertebral anterior y la siguiente respectivamente.

En referencia a la columna pélvica, sus vertebras están fusionadas y conforman dos huesos denominados sacro y coxis, con respecto al sacro es de forma triangular donde se fusionan 5 vertebras sacras (S1 a S5), en el borde superior está ubicada una masa central que conforma la parte más anterior del sacro y es llamado promontorio o ángulo sacro vertebral (Sierra et al., 2018). Así mismo, posee cuatro orificios que se ubican a cada lado del hueso sacro y a través de ellos pasan los nervios sacros, de igual manera en la zona lateral está ubicada la superficie articular que se une con la pelvis y conforma la zona de la articulación sacroilíaca.

La zona del coxis está conformada por cuatro vertebras fusionadas que forman un hueso triangular, su superficie es cóncava y tiene la capacidad de flexionarse y extenderse, estos movimientos permiten la defecación y también durante el parto (Sinchiguano, 2018).

De esta manera la columna vertebral también está conformada por ligamentos, articulaciones y sus discos intervertebrales (ver Figura 4), y es que dentro del organismo tiene un proceso complejo, que puede sufrir diversas afecciones musculo esqueléticas, y algunas de ellas degenerativas que afectan y limitan con el paso del tiempo la movilidad de tanto de la estructura como del organismo causando hasta rigidez, generando cambios morfológicos evidentes y una de estas afecciones son las espondilo artropatías (Plischuk \& Salceda, 2015).

\section{Figura 4}

\section{Disco Intervertebral}

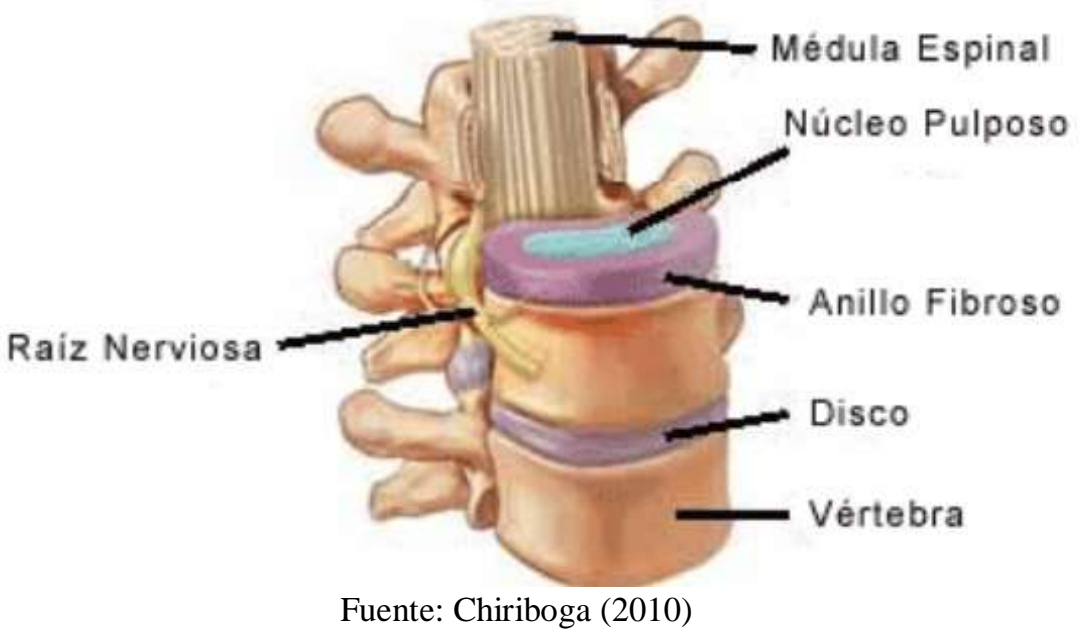

En este sentido, se menciona que una de las afecciones presentes en la columna vertebral es la espondilitis anquilosante, la cual es una artritis que se caracteriza por presentar 
inflamación crónica y las articulaciones se van degenerando, que afecta en gran proporción la columna vertebral y la articulación sacroilíaca (Torres, 2019), asimismo las vértebras afectadas por esta patología con el paso del tiempo se pueden unir.

Etimológicamente se menciona que según el diccionario médico de la Universidad de Salamanca citado por Manotoa (2019), se refiere a la inflamación de una o dos vértebras de allí que el origen de su nombre proviene de la palabra spondyl "vertebra" + itis "inflamación”. Se realizaron los primeros hallazgos de esta patología en el siglo XVII y XVIII en un cadáver, que, al verificar su estructura ósea, se notó que las articulaciones sacroilíacas, vertebras y apófisis estaban fusionadas.

Se desconocen las causas de forma precisa de esta afección, y se ha asociado a esta la presencia de un gen llamado HLA-B27, sin embargo, algunas personas poseen este gen y no desarrollan nunca la Espondilits Anquilosante, por lo tanto, no es concluyente que esta sea una causa especifica (López-Guillen, 2019).

Se han realizado estudios que determinan que existen una serie de factores de riesgo como la edad, el sexo y la genética, en este caso se indica que los hombres tienen mayores posibilidades de desarrollar esta patología, y aparece al final de la adolescencia o en la adultez temprana (López-Guillen, 2019).

La espondilitis anquilosante por ser una enfermedad autoinmune se han realizado numerosas investigaciones para poder determinar la fisiopatología de esta, considerando los factores genéticos, microbiota, ambientales y la posible existencia de anormalidades endocrinas (Manotoa, 2019).

En este sentido se muestra en la Tabla 1 una descripción de estas interacciones para tener más claro los factores que se estudian para determinar las razones de la existencia de esta enfermedad.

Tabla 1

Factores que se estudian para determinar las razones de la existencia de la EA

\begin{tabular}{ll}
\hline Interacciones & \multicolumn{1}{c}{ Descripción } \\
\hline & Hace más de 50 años que se realizó la primera idea para la relación genética, para \\
& revelar gradualmente 1 naturaleza poligénica. La probabilidad de riesgo de \\
& recurrencia relativa en familiares de primer grado o hermanos en comparación a la \\
& prevalencia de la población general es de 52-94, esto disminuye al aumentar la \\
Factor genético & distancia de relación familiar. Este riesgo es más recurrente en familiares de primer \\
& grado con HLA B27 positivo, en comparación con portadores de la población \\
& general. La EA está implicado con múltiples alelos HLA B con mayor riego en el \\
& HLA B27 13:02, 40:01, 40:02, 47:01 y 51:01 y con mejor riesgo en HLA B07:02 \\
& presentes en HLA B27 tanto positivo como negativo. \\
\hline
\end{tabular}


Tabla 1

Factores que se estudian para determinar las razones de la existencia de la EA (continuación)

\begin{tabular}{|c|c|}
\hline Interacciones & Descripción \\
\hline $\begin{array}{l}\text { Factor inmune y } \\
\text { genético }\end{array}$ & $\begin{array}{l}\text { Inmunidad intestinal: la clínica de la espondilitis anquilosante está asociada a la } \\
\text { enfermedad inflamatoria intestinal (EII), en la mucosa intestinal intervienen } \\
\text { genes para la activación de células linfoides intestinales y detección de bacterias } \\
\text { y una anormalidad en ellos se asocia a la EII }\end{array}$ \\
\hline $\begin{array}{l}\text { Aminopeptidasa } \\
\text { en EA }\end{array}$ & $\begin{array}{l}\text { Se ha demostrado genéticamente que intervienen tres aminopeptidasas, ERAP } 1 \\
\text { codifica el retículo endoplásmico, ERAP } 2 \text { y NPEPPS que codifica a la } \\
\text { puromicina, están relacionadas con la EA. Las proteínas codificadas por estos } \\
\text { genes es reducir los péptidos al tamaño apropiado para su presentación por el } \\
\text { HLA. Si se produce una alteración en los péptidos antigénicos, tiene un efecto en } \\
\text { la presentación del antígeno HLA B27 por el brazo de cadenas pesadas, la } \\
\text { activación de células T ayudadoras (TH17) y la implicación de receptores de } \\
\text { células asesinas, lo cual produce inestabilidad en la actividad enzimática de las } \\
\text { aminopeptidasas. }\end{array}$ \\
\hline $\begin{array}{l}\text { Factores } \\
\text { ambientales }\end{array}$ & $\begin{array}{l}\text { El tabaquismo eleva el riesgo de presentar enfermedades autoinmunes, } \\
\text { incrementando de manera importante el riesgo de enfermedades cardiovasculares } \\
\text { en pacientes con enfermedades reumáticas. Adicional a ello se ha observado que } \\
\text { los pacientes fumadores con espondiloartritis presentan más lesiones en sus } \\
\text { radiografías de columna que los que no fuman, se asocia el tabaquismo con la } \\
\text { aparición temprana de la enfermedad y empeoramiento de la calidad de vida. }\end{array}$ \\
\hline
\end{tabular}

Fuente: Manotoa (2019)

Asimismo, existe una serie de comorbilidades, esto debido a que esta enfermedad forma huesos como un intento que tiene el organismo para mejorarse y curarse generando la fusión de secciones de algunas vertebras lo que puede afectar la movilidad y hasta se puede afectar la caja torácica, además puede provocar inflamación ocular (uveítis) la cual es muy frecuente en la $\mathrm{AE}$, y genera dolor en los ojos, las personas son más sensibles a la luz y su visión puede tornarse borrosa, pueden aparecer fracturas por compresión, pues los huesos se afinan en algunas personas cuando la enfermedad está iniciando, y al presentar este debilitamiento vertebral pueden colapsar y causar fracturas, que pueden llegar a dar la medula espinal y los nervios. También puede provocar problemas a nivel cardíaco donde se ve afectada la aorta y esta al inflamarse puede causar que sus tamaños aumenten causando que la válvula aortica se distorsione y su funcionamiento puede verse afectado (López-Guillen, 2019).

En este sentido, a través de esta investigación se desea lograr realizar una revisión sistemática que permita describir las causas y el diagnóstico de la espondilitis anquilosante. 


\section{Metodología}

Con el propósito de lograr alcanzar el objetivo de esta investigación se realizó una revisión sistemática que según menciona Moreno et al. (2018), se caracteriza por:

Tener y describir el proceso de elaboración transparente y comprensible para recolectar, seleccionar, evaluar críticamente y resumir toda la evidencia disponible con respecto a la efectividad de un tratamiento, de un diagnostico o algún pronóstico, donde la diferencia radica principalmente en los estudios primarios que serán incluidos y evaluados. (p.184)

Se procedió a realizar la selección del material necesario que incluye artículos científicos y trabajos de grado indexados en bases de datos como Elsevier, Google académico, y Scielo, para la investigación considerando una serie de criterios de inclusión y exclusión que permitieron garantizar confiabilidad en esta investigación.

\section{Selección de la información:}

Se consideró como aspectos incluyentes para la elección de los temas la siguiente información:

- Idioma español

- De nivel científico, maestría, o doctoral

- Fecha de búsqueda del 2011 al 2021

- Relación con Espondilitis anquilosante en título, palabras clave, y objetivos

Se realizó la investigación considerando artículos y trabajos de grado realizados desde el 2011 hasta el año 2021, seleccionando los temas más adecuados, y considerando aspectos que excluyan aquellos temas que no contengan los criterios de inclusión anteriormente descritos, de esta manera se procedió a seleccionar 16 artículos y trabajos de grado para realizar el análisis correspondiente de 388 referencias halladas en las bases de datos anteriormente descritas.

\section{Resultados y Discusión}

Con base en los 16 documentos seleccionados que cumplían con los criterios de inclusión, se procede a realizar un análisis detallado con información que se obtuvo de cada uno de ellos para la revisión con el propósito de poder conocer los resultados de cada uno de los documentos y poder realizar un resumen acerca de las conclusiones analizadas (ver tabla 2). 
Tabla 2

Proceso de Análisis

\begin{tabular}{|c|c|c|c|c|c|}
\hline Fuente & Año & País & Título & Objetivo & Conclusiones Principales \\
\hline $\begin{array}{l}\text { Rodríguez- } \\
\text { Hernández } \\
\text { et al. }\end{array}$ & 2011 & México & $\begin{array}{l}\text { Baja densidad } \\
\text { mineral ósea } \\
\text { en espondilitis } \\
\text { anquilosante }\end{array}$ & $\begin{array}{l}\text { Evaluar la } \\
\text { frecuencia de } \\
\text { densidad } \\
\text { mineral ósea } \\
\text { baja y } \\
\text { osteoporosis en } \\
\text { EA }\end{array}$ & $\begin{array}{l}\text { La presencia de baja DMO en } \\
\text { pacientes con EA es mayor que en } \\
\text { la población en general; esto } \\
\text { favorece un deterioro estructural } \\
\text { óseo que lleva al desarrollo de } \\
\text { fracturas por osteoporosis, lo que } \\
\text { repercute en la funcionalidad y } \\
\text { calidad de vida de los pacientes, } \\
\text { por lo que su detección precoz y el } \\
\text { empleo de terapéutica eficaz } \\
\text { disminuirá las probabilidades de } \\
\text { este evento. }\end{array}$ \\
\hline
\end{tabular}

Todas estas hipótesis sobre la

\begin{tabular}{|c|c|c|c|c|c|}
\hline $\begin{array}{l}\text { Suárez et } \\
\text { al. }\end{array}$ & 2012 & Cuba & $\begin{array}{l}\text { Formación de } \\
\text { hueso nuevo y } \\
\text { espondilitis } \\
\text { anquilosante }\end{array}$ & $\begin{array}{l}\text { Demostrar } \\
\text { que en el } \\
\text { tratamiento } \\
\text { con anti TNF } \\
\text { se logra } \\
\text { controlar el } \\
\text { proceso } \\
\text { inflamatorio, }\end{array}$ & $\begin{array}{l}\text { formación de hueso nuevo } \\
\text { participando en la patogenia de la } \\
\text { EA se encuentran en estudio en los } \\
\text { últimos años, las cuales podría } \\
\text { proporcionar los medios necesarios } \\
\text { para la producción de nuevos } \\
\text { fármacos que no solo actúen sobre } \\
\text { la inflamación, sino también sobre } \\
\text { la formación de hueso nuevo en esta } \\
\text { enfermedad, que es el resultado que } \\
\text { evoluciona a la limitación funcional } \\
\text { y perdida de la calidad de vida. }\end{array}$ \\
\hline $\begin{array}{l}\text { González- } \\
\text { Rodríguez } \\
\text { et al. }\end{array}$ & 2013 & México & $\begin{array}{l}\text { Espondilitis } \\
\text { anquilosante. } \\
\text { Conceptos } \\
\text { Generales }\end{array}$ & $\begin{array}{l}\text { Conocer los } \\
\text { conceptos } \\
\text { generales de } \\
\text { la AE }\end{array}$ & $\begin{array}{l}\text { La EA es la segunda causa de } \\
\text { consulta en el servicio de } \\
\text { reumatología de un hospital de } \\
\text { segundo nivel. A pesar de ello, la } \\
\text { evaluación clínica de estos } \\
\text { pacientes sigue siendo un reto para } \\
\text { los médicos. Se debe llegar a un } \\
\text { diagnóstico de manera precoz a fin } \\
\text { de instaurar un tratamiento eficaz } \\
\text { que evite el desarrollo de una } \\
\text { discapacidad y/o un daño estructural } \\
\text { en las articulaciones afectadas, } \\
\text { porque el grupo de presentación es } \\
\text { de gente joven y productiva. En los } \\
\text { últimos años los inhibidores del } \\
\text { factor de necrosis tumoral han } \\
\text { mostrado un gran avance } \\
\text { terapéutico en esta entidad, }\end{array}$ \\
\hline
\end{tabular}

\section{la}


Tabla 2

Proceso de Análisis (continuación)

\begin{tabular}{|c|c|c|c|c|c|}
\hline Fuente & Año & País & Título & Objetivo & Conclusiones Principales \\
\hline $\begin{array}{l}\text { González- } \\
\text { Rodríguez } \\
\text { et al. }\end{array}$ & 2013 & México & $\begin{array}{l}\text { Espondilitis } \\
\text { anquilosante. } \\
\text { Conceptos } \\
\text { Generales }\end{array}$ & $\begin{array}{l}\text { Conocer los } \\
\text { conceptos } \\
\text { generales de } \\
\text { la AE }\end{array}$ & $\begin{array}{l}\text { con una mejora en la calidad de vida } \\
\text { de muchos pacientes resistentes a } \\
\text { los antiinflamatorios no esteroideos } \\
\text { (AINE). Los antagonistas del TNF- } \\
\alpha \text { son eficaces y seguros a largo } \\
\text { plazo, pero es necesario su uso } \\
\text { continuo para un adecuado control } \\
\text { sintomático }\end{array}$ \\
\hline
\end{tabular}

En el estudio del paciente con neurofibromatosis tipo 1 presenta síntomas generales con dolor lumbar inflamatorio crónico,

Espondilitis anquilosante y enfermedad de

Solís et al. $2014 \quad$ Cuba Von

Recklinhausen Una asociación infrecuente
Encontrar una respuesta etiopatogénica para la asociación de ambas enfermedades limitando su movilidad en todos los segmentos de su columna vertebral en base a estos síntomas se determinó que padece de EA, sin embargo, no se encontró algún indicio de asociación entre ambas afecciones. Se determina que debido a estas dos afecciones la calidad de vida del paciente se ve afectado de forma significativa, así como la de otras personas que padecen las mismas enfermedades. El estudio avanzado en el campo de la genética y la genómica permitirá poder conocer diversas variaciones genéticas en los individuos y como unos tienen mayor probabilidad de padecer la EA, y no solo predecir quienes la pueden padecer sino también encontrar maneras para prevenir diversos hábitos de vida que puedan generar la aparición de esta y otras enfermedades ayudando a mejorar la calidad de vida de las personas. 


\section{Tabla 2}

\section{Proceso de Análisis (Continuación)}

Porro et al. $2014 \quad$ Cuba

Guía para la rehabilitación de la espondilitis anquilosante
Crear una guía de rehabilitación para ser aplicado en pacientes con EA

De forma general el grupo de expertos en EA de la Sociedad internacional de espondiloartritis (acuerdo compilado de un $92 \%$ ) y las recomendaciones de la Liga Europea de Reumatología plantean que la rehabilitación es especialmente efectiva en la función física de estos pacientes.

El $100 \%$ coincide que la terapia debe incluir ejercicios, así como un $98 \%$ refiere la utilidad de la educación e instrucciones, datos corroborados en nuestra experiencia, a través del seguimiento sistemático de los pacientes con EA que se atienden por nuestro servicio.

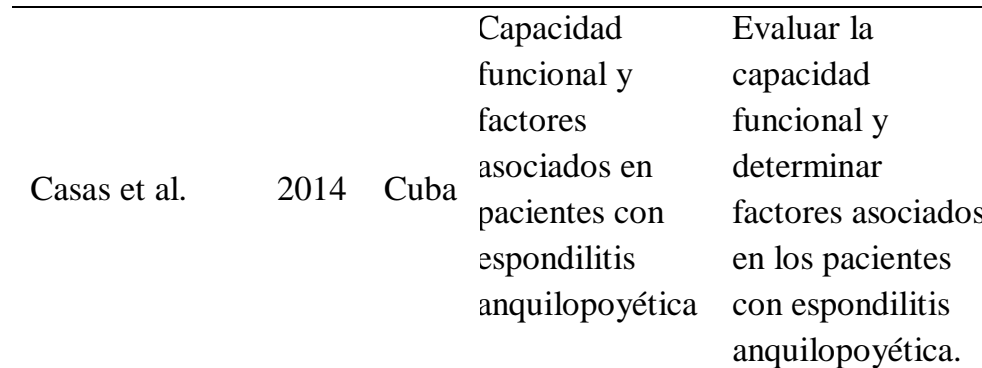

La capacidad funcional se asocia de forma significativa con la edad de inicio de la enfermedad, el tiempo de demora del diagnóstico, la afectación del segmento cervical, la ocupación laboral, la sacroilitis y la actividad de la enfermedad.

El paciente evaluado en esta investigación presenta síntomas generales, con presencia de dolor

Solís et al. (Espondilitis Anquilosante y Síndrome de Klippel-Feil. 2015 Cuba Una asociación infrecuente)
Espondilitis

Anquilosante y Síndrome de KlippelFeil. Una asociación infrecuente
Determinar la asociación existente entre el síndrome de Kliper-Feil y la Espondilitis anquilosante lumbar inflamatorio crónico, entensopatías y limitación de la movilidad de todos los segmentos de la columna vertebral, a través de estos síntomas se pudo diagnosticar al paciente con espondilitis anquilosante y no se evidencia la asociación de ambas afecciones ni relación mediante mecanismos etiopatogénicos que expliquen la presencia de ambas enfermedades. Caso interesante de EA por la

Espondilitis anquilosante Arevalo et al. $2016 \quad \begin{aligned} & \text { Méxi } \\ & \text { co }\end{aligned} \quad \begin{aligned} & \text { en mujer con } \\ & \text { en }\end{aligned}$ inicio en edad tardía

\section{Estudiar el} desarrollo de la EA en mujer con inicio en edad tardía presentación tardía y en mujer, ya que la enfermedad afecta predominantemente a hombres e inicia en la juventud. 
Tabla 2

Proceso de Análisis (Continuación)

\begin{tabular}{|c|c|c|c|c|c|}
\hline Fuente & Año & País & Título & Objetivo & Conclusiones Principales \\
\hline $\begin{array}{l}\text { Arevalo et } \\
\text { al. }\end{array}$ & 2016 & México & $\begin{array}{l}\text { Espondilitis } \\
\text { anquilosante en } \\
\text { mujer con } \\
\text { inicio en edad } \\
\text { tardía }\end{array}$ & $\begin{array}{l}\text { Estudiar el } \\
\text { desarrollo de } \\
\text { la EA en } \\
\text { mujer con } \\
\text { inicio en edad } \\
\text { tardía }\end{array}$ & $\begin{array}{l}\text { El desarrollo de terapias efectivas } \\
\text { para la EA, en particular, los } \\
\text { medicamentos biológicos } \\
\text { antifactor de necrosis tumoral alfa, } \\
\text { han logrado la mejoría de los } \\
\text { síntomas, la capacidad funcional y } \\
\text { la calidad de vida de muchos } \\
\text { pacientes. }\end{array}$ \\
\hline $\begin{array}{l}\text { Arevalo et } \\
\text { al. }\end{array}$ & 2016 & México & $\begin{array}{l}\text { Espondilitis } \\
\text { anquilosante en } \\
\text { mujer con } \\
\text { inicio en edad } \\
\text { tardía }\end{array}$ & $\begin{array}{l}\text { Estudiar el } \\
\text { desarrollo de } \\
\text { la EA en } \\
\text { mujer con } \\
\text { inicio en edad } \\
\text { tardía }\end{array}$ & $\begin{array}{l}\text { Los estudios clínicos y } \\
\text { radiográficos sugieren que pueden } \\
\text { retardar las secuelas esqueléticas, } \\
\text { aunque los reportes son } \\
\text { contradictorios. Por el momento, } \\
\text { hay escasa información del uso de } \\
\text { medicamentos anti-TNF alfa en } \\
\text { individuos con EA de inicio en } \\
\text { edad avanzada. Es bien conocida la } \\
\text { mayor incidencia de infecciones, } \\
\text { incluyendo tuberculosis, y de } \\
\text { trastornos mieloproliferativos, por } \\
\text { lo que su administración amerita } \\
\text { especial cuidado en este grupo de } \\
\text { enfermos. } \\
\text { Al igual que lo referido en la } \\
\text { literatura, los FARME fallaron en } \\
\text { controlar las manifestaciones } \\
\text { axiales de la enfermedad, lo que } \\
\text { ameritó el uso de etanercept. En } \\
\text { nuestro caso la eficacia y tolerancia } \\
\text { al etanercept fue tan buena como la } \\
\text { que se obtiene en los jóvenes, pero } \\
\text { se requiere realizar estudios en } \\
\text { casos semejantes al descrito para } \\
\text { normar la conducta a seguir. La } \\
\text { buena respuesta obtenida con } \\
\text { etanercept es debido a que éste } \\
\text { actúa como un receptor de la } \\
\text { citocina inflamatoria factor de } \\
\text { necrosis tumoral } \\
\text { bloqueándola e inhibiendo de esta } \\
\text { forma la respuesta inflamatoria. El } \\
\text { FNT ha sido encontrado en las } \\
\text { articulaciones inflamadas de } \\
\text { pacientes con EA. }\end{array}$ \\
\hline
\end{tabular}


Tabla 2

Proceso de Análisis (Continuación)

\begin{tabular}{|c|c|c|c|c|c|}
\hline Fuente & Año & País & Título & Objetivo & Conclusiones Principales \\
\hline Delgado & 2016 & España & $\begin{array}{l}\text { Bases } \\
\text { temperamentale } \\
\text { s del sentido del } \\
\text { humor y } \\
\text { actividad de la } \\
\text { enfermedad en } \\
\text { pacientes con } \\
\text { espondilitis } \\
\text { anquilosante y } \\
\text { artritis } \\
\text { reumatoide }\end{array}$ & $\begin{array}{l}\text { contribuir desde } \\
\text { una } \\
\text { aproximación } \\
\text { psicológica a un } \\
\text { mejor } \\
\text { entendimiento } \\
\text { de la influencia } \\
\text { que tienen } \\
\text { algunos de } \\
\text { factores } \\
\text { psicológicos sin } \\
\text { explorar sobre } \\
\text { el estado de } \\
\text { salud que tienen } \\
\text { pacientes con } \\
\text { espondilitis } \\
\text { anquilosante y } \\
\text { artritis } \\
\text { reumatoide }\end{array}$ & $\begin{array}{l}\text { Se evidencia la relación de factores } \\
\text { emocionales negativos (estrés, la } \\
\text { ansiedad y depresión) como } \\
\text { positiva } \text { (problemas } \\
\text { cardiovasculares, quejas } \\
\text { somáticas, relaciones sociales y } \\
\text { dolor crónico) Contexto de estudio } \\
\text { en el que habría que situar una de } \\
\text { las variables psicológicas } \\
\text { estudiadas en esta tesis doctoral, es } \\
\text { decir la alegría estado-rasgo y sus } \\
\text { posibles efectos beneficiosos ante } \\
\text { determinadas patologías } \\
\text { procesos de salud. Tal y como se } \\
\text { viene insistiendo en la presente } \\
\text { tesis doctoral, la alegría está } \\
\text { relacionada con un afrontamiento } \\
\text { más eficiente de los problemas, a } \\
\text { la vez que una mayor facilidad } \\
\text { para recobrar el equilibrio ante } \\
\text { circunstancias difíciles }\end{array}$ \\
\hline $\begin{array}{l}\text { Sanhueza } \\
\text { et al. }\end{array}$ & 2016 & Chile & $\begin{array}{l}\text { Espondilitis } \\
\text { anquilosante: } \\
\text { revisión de } \\
\text { hallazgos } \\
\text { imagenológicos } \\
\text { en la columna }\end{array}$ & $\begin{array}{l}\text { Realizar una } \\
\text { revisión } \\
\text { pictográfica de } \\
\text { hallazgos } \\
\text { imagenológicos } \\
\text { presentes en la } \\
\text { columna } \\
\text { vertebral de } \\
\text { pacientes } \\
\text { portadores de } \\
\text { EA. }\end{array}$ & $\begin{array}{l}\text { La EA se caracteriza por típicas } \\
\text { manifestaciones en RM, sin } \\
\text { embargo, estas no son específicas } \\
\text { y pueden verse en múltiples otras } \\
\text { patologías de columna. Es de } \\
\text { utilidad tener en cuenta que los } \\
\text { cambios inflamatorios de la } \\
\text { columna en patologías } \\
\text { inflamatorias no ocurren de forma } \\
\text { aislada y están asociados en su } \\
\text { mayoría a afección de } \\
\text { articulaciones sacroilíacas, } \\
\text { teniendo características } \\
\text { imagenológicas clave en sitios } \\
\text { específicos y una evolución } \\
\text { determinada en el tiempo. En } \\
\text { pacientes con historia de dolor } \\
\text { referido a la columna vertebral } \\
\text { inespecífico que son evaluados } \\
\text { con imágenes es importante } \\
\text { clasificar correctamente los } \\
\text { hallazgos, para iniciar otros test } \\
\text { diagnósticos correspondientes }\end{array}$ \\
\hline
\end{tabular}


Tabla 2

Proceso de Análisis (Continuación)

\begin{tabular}{|c|c|c|c|c|c|}
\hline Fuente & Año & País & Título & Objetivo & Conclusiones Principales \\
\hline $\begin{array}{l}\text { Andaluz } \\
\text { et al. }\end{array}$ & 2017 & Cuba & $\begin{array}{l}\text { Afecciones } \\
\text { oftalmológicas } \\
\text { más } \\
\text { frecuentemente } \\
\text { asociadas a } \\
\text { enfermedades } \\
\text { reumáticas }\end{array}$ & $\begin{array}{l}\text { revisar las } \\
\text { afecciones } \\
\text { oftalmológicas } \\
\text { más } \\
\text { frecuentemente } \\
\text { asociadas a } \\
\text { enfermedades } \\
\text { reumática }\end{array}$ & $\begin{array}{l}\text { En resumen, las enfermedades } \\
\text { reumáticas son frecuentemente } \\
\text { infravaloradas en las distintas } \\
\text { estrategias de atención al enfermo } \\
\text { crónico, pues los esfuerzos se } \\
\text { enfocan en las patologías que } \\
\text { exhiben mayor mortalidad, } \\
\text { minimizando entonces la } \\
\text { discapacidad, fuente de gran coste } \\
\text { socio sanitario. Es fundamental } \\
\text { que se profundice en la } \\
\text { comprensión y manejo de aspectos } \\
\text { esenciales presentes en la relación } \\
\text { entre enfermedades reumáticas y } \\
\text { las diversas manifestaciones } \\
\text { oftalmológicas, ya que el } \\
\text { diagnóstico correcto de estas, } \\
\text { puede de hecho ayudar a destacar } \\
\text { el rol del proceso sistémico y } \\
\text { viceversa: en las personas que } \\
\text { sufren un proceso reumático con } \\
\text { afectación ocular, no se resolverá } \\
\text { el problema oftalmológico si no se } \\
\text { trata correctamente la ER. Resulta } \\
\text { indispensable desarrollar } \\
\text { estrategias de prevención } \\
\text { destinadas a disminuir la } \\
\text { morbilidad asociada a las ER y es } \\
\text { necesario trabajo } \\
\text { multidisciplinario que garantice la } \\
\text { adecuada evaluación, tratamiento } \\
\text { y seguimiento de estas } \\
\text { manifestaciones, contando con la } \\
\text { participación conjunta de } \\
\text { reumatólogos y oftalmólogos. } \\
\text { El tratamiento precoz, tras un } \\
\text { acertado diagnóstico de las } \\
\text { afecciones oculares, contribuye a } \\
\text { mejorar el pronóstico visual del } \\
\text { paciente. }\end{array}$ \\
\hline
\end{tabular}


Tabla 2

Proceso de Análisis (Continuación)

\begin{tabular}{|c|c|c|c|c|c|}
\hline Fuente & Año & País & Título & Objetivo & Conclusiones Principales \\
\hline $\begin{array}{c}\text { Andaluz et } \\
\text { al. }\end{array}$ & 2017 & Cuba & $\begin{array}{c}\text { Afecciones } \\
\text { oftalmológicas } \\
\text { más } \\
\text { frecuentemente } \\
\text { asociadas a } \\
\text { enfermedades } \\
\text { reumáticas }\end{array}$ & $\begin{array}{l}\text { revisar las } \\
\text { afecciones } \\
\text { oftalmológicas } \\
\text { más } \\
\text { frecuentemente } \\
\text { asociadas a } \\
\text { enfermedades } \\
\text { reumática } \\
\end{array}$ & $\begin{array}{l}\text { Las manifestaciones } \\
\text { oftalmológicas requieren ser } \\
\text { evaluadas para su diagnóstico y } \\
\text { tratamiento oportuno, ya que } \\
\text { pueden ser causa de ceguera y se } \\
\text { presentan acompañando } \\
\text { enfermedades sistémicas de } \\
\text { reconocida morbimortalidad }\end{array}$ \\
\hline $\begin{array}{l}\text { Fernández } \\
\text { et al. }\end{array}$ & 2018 & Cuba & $\begin{array}{c}\text { Análisis de un } \\
\text { caso de } \\
\text { espondilitis } \\
\text { anquilopoyética }\end{array}$ & $\begin{array}{c}\text { Mostrar con la } \\
\text { presentación del } \\
\text { caso el } \\
\text { tratamiento de un } \\
\text { paciente afectado } \\
\text { con espondilitis } \\
\text { anquilopoyética, } \\
\text { toma de } \\
\text { articulaciones } \\
\text { periféricas y el } \\
\text { resultado obtenido } \\
\text { con la cirugía } \\
\text { correctora y la } \\
\text { rehabilitación. }\end{array}$ & $\begin{array}{l}\text { La cirugía reparadora y las } \\
\text { modalidades fisioterapéuticas } \\
\text { utilizadas en el paciente } \\
\text { mostraron ser eficaces. La } \\
\text { hidroterapia y el ejercicio fueron } \\
\text { los pilares fundamentales en la } \\
\text { rehabilitación. Se mejoró la } \\
\text { calidad de vida y la autoestima } \\
\text { del paciente, incluso se logró su } \\
\text { reinserción socio-laboral. }\end{array}$ \\
\hline $\begin{array}{c}\text { Di } \\
\text { Bonavent } \\
\text { ura }\end{array}$ & 2019 & España & $\begin{array}{c}\text { Espondilitis } \\
\text { anquilosante, } \\
\text { ayuno } \\
\text { intermitente, } \\
\text { probióticos y } \\
\text { ejercicio } \\
\text { anaeróbico: una } \\
\text { revisión } \\
\text { sistemática } \\
\text { sobre las formas } \\
\text { de tratamiento } \\
\text { más } \\
\text { actualizadas }\end{array}$ & $\begin{array}{c}\text { Evaluar la } \\
\text { efectividad del } \\
\text { empleo de ayuno } \\
\text { intermitente, } \\
\text { probióticos y de } \\
\text { los ejercicios de } \\
\text { tipo anaeróbico en } \\
\text { términos de } \\
\text { funcionalidad, } \\
\text { movilidad, } \\
\text { actividad de la } \\
\text { enfermedad y } \\
\text { calidad de vida en } \\
\text { pacientes con EA. }\end{array}$ & $\begin{array}{l}\text { Teniendo en cuenta el impacto } \\
\text { negativo significativo de la EA } \\
\text { en la funcionalidad y la calidad } \\
\text { de vida, es fundamental } \\
\text { investigar sobre las modalidades } \\
\text { potencialmente efectivas en el } \\
\text { tratamiento del paciente con } \\
\text { espondilitis anquilosante. Según } \\
\text { los resultados de la presente } \\
\text { investigación, el entrenamiento } \\
\text { de tipo anaeróbico tiene un } \\
\text { efecto positivo no solo sobre la } \\
\text { disminución de los síntomas de } \\
\text { la enfermedad (dolor, rigidez, } \\
\text { fatiga), sino también sobre los } \\
\text { parámetros fisiológicos (VO2, } \\
\text { VSG) y psicológicos (ansiedad, } \\
\text { nivel de angustia). }\end{array}$ \\
\hline
\end{tabular}


Tabla 2

Proceso de Análisis (Continuación)

\begin{tabular}{|c|c|c|c|c|c|}
\hline Fuente & Año & País & Título & Objetivo & Conclusiones Principales \\
\hline $\begin{array}{l}\text { Di } \\
\text { Bonavent } \\
\text { ura }\end{array}$ & 2019 & España & $\begin{array}{c}\text { Espondilitis } \\
\text { anquilosante, } \\
\text { ayuno } \\
\text { intermitente, } \\
\text { probióticos y } \\
\text { ejercicio } \\
\text { anaeróbico: una } \\
\text { revisión } \\
\text { sistemática } \\
\text { sobre las formas } \\
\text { de tratamiento } \\
\text { más } \\
\text { actualizadas }\end{array}$ & $\begin{array}{c}\text { Evaluar la } \\
\text { efectividad del } \\
\text { empleo de } \\
\text { ayuno } \\
\text { intermitente, } \\
\text { probióticos y de } \\
\text { los ejercicios de } \\
\text { tipo anaeróbico } \\
\text { en términos de } \\
\text { funcionalidad, } \\
\text { movilidad, } \\
\text { actividad de la } \\
\text { enfermedad y } \\
\text { calidad de vida } \\
\text { en pacientes con } \\
\text { EA. }\end{array}$ & $\begin{array}{l}\text { En cuanto al uso de probióticos, } \\
\text { a pesar de la estrecha relación } \\
\text { entre microbiota y EA, aún no se } \\
\text { dispone de una evidencia solida } \\
\text { que justifique su } \\
\text { implementación. Resultados } \\
\text { similares se encuentran para el } \\
\text { ayuno intermitente. Cabe } \\
\text { destacar que las mejorías que } \\
\text { emergen de los estudios } \\
\text { analizados, evidencian un } \\
\text { posible papel relevante de estos } \\
\text { tratamientos en la disminución } \\
\text { de la inflamación crónica y de } \\
\text { los altos niveles de glucosa sobre } \\
\text { el cartílago y su degeneración en } \\
\text { pacientes con Espondilitis } \\
\text { Anquilosante. Para finalizar, el } \\
\text { presente estudio agrega valor } \\
\text { científico a la evidencia actual en } \\
\text { comparación con las revisiones } \\
\text { publicadas anteriormente, ya que } \\
\text { es una revisión sistemática que } \\
\text { compara diferentes tipos de } \\
\text { tratamiento para la misma } \\
\text { patología. }\end{array}$ \\
\hline $\begin{array}{l}\text { Montiel- } \\
\text { Esparza } \\
\text { et al. }\end{array}$ & 2019 & México & $\begin{array}{l}\text { Arteritis de } \\
\text { Takayasu con } \\
\text { espondilitis } \\
\text { anquilosante }\end{array}$ & $\begin{array}{l}\text { Evaluar la } \\
\text { asociación } \\
\text { existente entre } \\
\text { la arteritis de } \\
\text { Takayasu y la } \\
\text { espondilitis } \\
\text { anquilosante }\end{array}$ & $\begin{array}{l}\text { Como causa de deceso se llegó a } \\
\text { la conclusión de muerte súbita } \\
\text { ocasionada por la hipertrofia } \\
\text { miocárdica abunda a la estenosis } \\
\text { de la aorta ocasionada por la } \\
\text { arteritis de Takayasu que padecía } \\
\text { el paciente, en este caso no se } \\
\text { encontraron malformaciones } \\
\text { cardiacas concomitantes con } \\
\text { espondilitis anquilosante. }\end{array}$ \\
\hline
\end{tabular}


Tabla 2

Proceso de Análisis (Continuación)

\begin{tabular}{|c|c|c|c|c|c|}
\hline Fuente & Año & País & Título & Objetivo & Conclusiones Principales \\
\hline $\begin{array}{l}\text { Montiel- } \\
\text { Esparza } \\
\text { et al. }\end{array}$ & 2019 & México & $\begin{array}{l}\text { Arteritis de } \\
\text { Takayasu con } \\
\text { espondilitis } \\
\text { anquilosante }\end{array}$ & $\begin{array}{l}\text { Evaluar la } \\
\text { asociación } \\
\text { existente entre } \\
\text { la arteritis de } \\
\text { Takayasu y la } \\
\text { espondilitis } \\
\text { anquilosante }\end{array}$ & $\begin{array}{l}\text { La arteritis de Takayasi no } \\
\text { siempre es una enfermedad } \\
\text { aislada, asimismo, la espondilitis } \\
\text { anquilosante, si bien se ha } \\
\text { relacionado, con muchas } \\
\text { enfermedades autoinmunitarias, } \\
\text { puede simular síntomas de } \\
\text { arteritis de Takayasu como } \\
\text { diagnostico agregado en sujetos } \\
\text { con espondilitis. } \\
\text { El paciente en cuestión que } \\
\text { infortunadamente } \\
\text { demuestra una vez más la } \\
\text { necesidad de realizar un } \\
\text { concienzudo examen en los } \\
\text { pacientes. Asimismo, se sugiere } \\
\text { que ante el nuevo sistema penal } \\
\text { acusatorio y ante una muerte } \\
\text { súbita se efectúe durante la } \\
\text { necropsia la tinción con azul de } \\
\text { tetrazoilo que ayudara a } \\
\text { distinguir entre afección } \\
\text { miocárdica por infractor agudo } \\
\text { de miocardio y otra enfermedad } \\
\text { de repercusión miocárdica como } \\
\text { el caso comunicado a quien no se } \\
\text { le efectúo tal prueba. }\end{array}$ \\
\hline $\begin{array}{l}\text { Bazán et } \\
\text { al. }\end{array}$ & 2021 & $\begin{array}{l}\text { Argenti } \\
\text { na }\end{array}$ & $\begin{array}{l}\text { Fractura de la } \\
\text { columna } \\
\text { vertebral en } \\
\text { pacientes con } \\
\text { espondilitis } \\
\text { anquilosante }\end{array}$ & $\begin{array}{l}\text { Considerar las } \\
\text { dificultades } \\
\text { diagnósticas, } \\
\text { describir las } \\
\text { lesiones y la } \\
\text { decisión } \\
\text { terapéutica, } \\
\text { analizar la } \\
\text { presentación de } \\
\text { complicaciones } \\
\text { y realizar una } \\
\text { actualización } \\
\text { bibliográfica }\end{array}$ & $\begin{array}{l}\text { Los pacientes con EA corren } \\
\text { más riesgo de sufrir una fractura } \\
\text { vertebral por traumatismo de } \\
\text { baja energía que la población } \\
\text { general. Es importante tener un } \\
\text { alto nivel de sospecha ante un } \\
\text { paciente con EA y un } \\
\text { antecedente traumático que solo } \\
\text { sufra dolor, para evitar las } \\
\text { complicaciones neurológicas. En } \\
\text { nuestro estudio, el promedio de } \\
\text { días hasta el diagnóstico fue } 12 \text { y } \\
\text { se relacionó con la falta de } \\
\text { estudios o la mala calidad de } \\
\text { estos. }\end{array}$ \\
\hline
\end{tabular}




\section{Tabla 2}

Proceso de Análisis (Continuación)

\begin{tabular}{|c|c|c|c|c|c|}
\hline Fuente & Año & País & Título & Objetivo & Conclusiones Principales \\
\hline $\begin{array}{l}\text { Bazán et } \\
\text { al. }\end{array}$ & 2021 & $\begin{array}{l}\text { Argenti } \\
\text { na }\end{array}$ & $\begin{array}{l}\text { Fractura de la } \\
\text { columna } \\
\text { vertebral en } \\
\text { pacientes con } \\
\text { espondilitis } \\
\text { anquilosante }\end{array}$ & $\begin{array}{l}\text { Considerar las } \\
\text { dificultades } \\
\text { diagnósticas, } \\
\text { describir las } \\
\text { lesiones y la } \\
\text { decisión } \\
\text { terapéutica, } \\
\text { analizar la } \\
\text { presentación de } \\
\text { complicaciones } \\
\text { y realizar una } \\
\text { actualización } \\
\text { bibliográfica }\end{array}$ & $\begin{array}{l}\text { En esta serie, el mecanismo de } \\
\text { lesión más frecuente fue la } \\
\text { hiperflexión con la consiguiente } \\
\text { lesión de la banda de tensión } \\
\text { posterior asociada o no con } \\
\text { traslación. Los antiinflamatorios } \\
\text { no esteroides brindarían un } \\
\text { efecto protector frente al riesgo } \\
\text { de sufrir una fractura vertebral } \\
\text { en pacientes con EA. El } \\
\text { tratamiento quirúrgico con } \\
\text { fijaciones largas y liberación por } \\
\text { vía posterior es el más utilizado. } \\
\text { En nuestra serie, no se } \\
\text { observaron complicaciones } \\
\text { postquirúrgicas. Se cree } \\
\text { necesaria una muestra con más } \\
\text { pacientes }\end{array}$ \\
\hline
\end{tabular}

Fuente: Autor (2022)

En las investigaciones realizadas por Rodríguez-Hernández et al. (2011), Suárez et al. (2012) y Bazán et al. (2021), mencionan que el deterioro óseo en los pacientes con EA genera mayor porcentaje de fracturas por osteoporosis en comparación con la población en general, y que al detectar a tiempo la enfermedad podría evitarse este tipo de acontecimientos que entorpece la calidad de vida del paciente, e igualmente estas evidencias podrían proporcionar información importante acerca del comportamiento de esta enfermedad con el propósito de producir nuevos medicamentos que no solamente alivien los procesos inflamatorios sino también evitar la formación de hueso nuevo en las fusiones existentes así como evitar fracturas que podría permitir una mejor calidad de vida de los pacientes.

De la misma manera González-Rodríguez et al. (2013) coincide en que la detección temprana de la EA mejorara en gran medida la calidad de vida de los pacientes, que cada día es más común la existencia de ellos en los centros de reumatología, es importante poder detectar a tiempo esta enfermedad para indicar un tratamiento acorde a las necesidades de los pacientes que disminuya su discapacidad y un posible daño estructural en sus articulaciones.

Los autores Solís et al. (2014), Solís et al. (2015), Delgado (2016), Andaluz et al. (2017) y Montiel-Esparza et al. (2019), determinan que la EA puede estar presente con otras patologías, como afecciones oftalmológicas, artritis psoriásica y reumatoide, Síndrome 
de Klippel-Feil y Arteritis de Takayasu, unas más comunes que otras, sin embargo además de afectar el organismo del individuo también compromete el estado psicológico de las personas pues estas enfermedades o síndromes afectan la vida cotidiana, su vida laboral y la interacción social lo que genera estrés y depresión en estas personas.

Asimismo Porro et al. (2014), Sanhueza et al. (2016) y Di Bonaventura (2019), establecen en sus investigaciones que esta enfermedad puede ser diagnosticada a través de imagenología, (RX, RM) además de exámenes de laboratorio y exámenes físicos donde se determinan la forma de la columna y otras complicaciones que pueden estar presentes en el cuerpo de las personas, donde además de tratamiento médico con fármacos la rehabilitación física es una terapia efectiva donde se deben incluir ejercicios anaeróbicos que ayudan a disminuir los síntomas de dolor, rigidez, fatiga entre otros, y mejora los niveles de estrés, angustia y ansiedad.

De la misma manera Castro-Santos et al. (2014), muestra en su investigación que la EA está asociada a alteraciones genéticas con presencia positiva del HLA B27 aunque no necesariamente todas las personas que estén positivas en este gen manifiesten síntomas existe evidencia de la presencia de EA en un gran porcentaje de individuos, estos avances en los estudios genéticos permitirán analizar y poder prevenir diversos hábitos de vida que generan la aparición de esta enfermedad y de muchas otras enfermedades que dará paso a la mejora de la calidad de vida de estos pacientes.

\section{Conclusiones}

- Es evidente que existe una gran variedad de afecciones que pueden estar asociadas con la EA, sin embargo, se observa la coincidencia en que la mayoría de los autores analizados manifiestan que una de las principales consecuencias que genera esta enfermedad es el deterioro de la calidad de vida de los individuos que padecen esta y otras enfermedades durante su vida, y que repercuten en el comportamiento psicológico de los mismos.

- Es importante destacar que se debe seguir realizando análisis y estudios genéticos y de casos de pacientes con esta patología que permita determinar de forma más clara las causas que generan esta enfermedad y la prevalencia de la misma en los individuos, así como poder determinar posibles nuevos tratamientos que permitan mejorar en gran medida los síntomas, como el dolor, la inflamación y la rigidez para que las personas con esta enfermedad puedan realizar sus actividades sin depender de la ayuda de otras personas. 


\section{Referencias Bibliográficas}

Andaluz, M., Cifuentes, M., Dávila, E., \& Reyes, L. (2017). Afecciones oftalmológicas más frecuentemente asociadas a enfermedades reumáticas. Revista Cubana de Reumatología 19(3), 202-208.

Arevalo, F., Badia, J., \& Arciniega, A. (2016). Espondilitis anquilosante en mujer con inicio en edad tardía. Acta Médica Grupo Ángeles 14(2), 108-110.

Bazán, P., Bravo, M., Gutiérrez, E., Terraza, S., Cortés, C., Borri, Á. M., y otros. (2021). Fractura de la columna vertebral en pacientes con espondilitis anquilosante. Rev Asoc Argent Ortop Traumatol 86(1), 58-63.

Casas, N., Vidal, J., Pérez, D., Castell, C., \& Gutiérrez, Á. (2014). Capacidad funcional y factores asociados en pacientes con espondilitis anquilopoyética. Revista Cubana de Medicina 53(1), 50-60.

Castro-Santos, P., Gutiérrez, M., \& Díaz-Peña, R. (2014). Genética HLA-B27 y espondilitis anquilosante: 40 años. Rev. Med. Chile 142, 1165-1173.

Chiriboga, A. (2010). Enfermedades músculo esqueléticas de la columna vertebral mas frecuentes atentidas en el área de rehabilitación física de la Cruz Roja Ecuatoriana junta provincial de Pichincha, sede Norte durante el año 2008. Quito, Ecuador: Trabajo especial de grado de la Pontificia Universidad Católica del Ecuador para optar al título de Licenciatura en Terapia Física.

Delgado, C. (2016). Bases temperamentales del sentido del humor y actividad de la enfermedad en pacientes con espondilitis anquilosante y artritis reumatoide. Trabajo especial de grado de la Universidad D Córdoba.

Di Bonaventura, S. (2019). Espondilitis anquilosante, ayuno intermitente, probióticos y ejercicio anaeróbico: una revisión sistemática sobre las formas de tratamiento más actualizadas. España: Trabajo especial de grado de la Universidad Camilo José Cela.

Espinoza, L. (2017). Sistema para monitorear el grado de curvatura de la columna vertebral, mediante la utilización de sensores IMU. Riobamba, Ecuador: Trabajo especial de grado de la Escuela Superior Politécnica de Chimborazo para optar al título de Ingeniería en Electrónica Control y Redes Industriales.

Fernández, L., Heredia, Y, Chacón, M., \& Coronado, Y. (2018). Análisis de un caso de espondilitis anquilopoyética. Revista Cubana de Medicina Física y Rehabilitación 10(3), $1--10$. 
González-Rodríguez, M., Guerrero-Soto, A., Corona-Sánchez, E., Rocha-Muñoz, A., Díaz-González, E., \& González-López, L. (2013). Espondilitis anquilosante. Conceptos Generales. El Residente 883), 106-113.

López-Guillen, A. (2019). Evaluación del menoscabo por Espondilitis Anquilosante. Unión Pericial Sanitaria.

Manotoa, M. (2019). Evaluar calidad de vida y funcionalidad en pacientes con espondilitis anquilosante. Quito, Ecuador: Trabajo especial de grado de la Pontificia Universidad Católica del Ecuador para optar al título de Especialista en Medicina Interna.

Montiel-Esparza, A., Sosa-Morales, V., Bullón-Alcalá, S., \& Zavaleta-Martínez, M. (2019). Arteritis de Takayasu con espondilitis anquilosante. Med. Int. Méx 35(3), 448-453.

Moreno, B., Muñoz, M., Cuellar, J., Domancic, S., \& Villanueva, J. (2018). Metodología. Revisiones sistemáticas: definición y nociones básicas. Rev. Clin. Periodoncia Implantol. Rehabil. Oral 11(3), 184-186.

Plischuk, M., \& Salceda, S. (2015). Espondilitis anquilosante en una población contemporánea de La Plata, Argentina. Rev. Esp. Antrop. Fís. 36, 22-32.

Porro, J., Estévez, A., Rodríguez, A., Suárez, R., \& González, B. (2014). Guía para la rehabilitación de la espondilitis anquilosante. Revista Cubana de Reumatología XVI (2), 245-252.

Rodríguez, M., \& Pérez, N. (2021). Manual de anatomía humana normal. Chile: Universidad Andrés Bello 2da edición.

Rodríguez-Hernández, D., Villalpando-Gómez, Y., De la Cerda-Trujillo, L., RochaMuñoz, A., Fuentes-González, C., Moreno-Sandoval, I., y otros. (2011). Baja densidad mineral ósea en espondilitis anquilosante. El Residente VI (1), 14-19.

Sanhueza, A., Prieto, J., Weisz, J., Leiter, F., Soto, S. F., \& Lira, L. (2016). Espondilitis anquilosante: revisión de hallazgos imagenológicos en la columna. Revista Chilena de Radiología 22(4), 171-183.

Sierra, A; Lozano, L; Dávila, C; Mora, J; Tramontini, C. (2018). Anatomía de la Columna Vertebral en radiografía convencional. Revista Medica Sanitas 21(1), 39-46.

Sinchiguano, A. (2018). Caracterización de patologías músculo-esqueléticas de la columna vertebral en usuarios de 18 a 65 años del centro de rehabilitación física en la facultad de educación física de la Universidad Central del Ecuador durante 
el período enero-diciembre 2017. Quito, Ecuador: Trabajo especial de grado de la Universidad Central del Ecuador para optar al título de Licenciado en Terapia Física.

Solís, U., Morejón, J., \& de Armas, A. (2014). Espondilitis anquilosante y enfermedad de Von Recklinhausen. Una asociación infrecuente. Revista Cubana de Reumatología XVI (2), 240-244.

Solís, U., Morejón, J., de Armas, A., Prada, D., \& Amador, D. (2015). Espondilitis Anquilosante y Síndrome de Klippel-Feil. Una asociación infrecuente. Revista Cubana de Reumatología XVII (2), 206-210.

Suárez, R., Molinero, C., Prada, D., López, A., \& Lemagne, Y. (2012). Formación de hueso nuevo y espondilitis anquilosante. Revista Cubana de Reumatología XIV (20), 1-6.

Torres, A. (2019). Metaanálisis sobre la efectividad de la realización de ejercicio físico en pacientes con Espondilitis Anquilosante. Archives of Nursing Research, 3(1), 1-18.

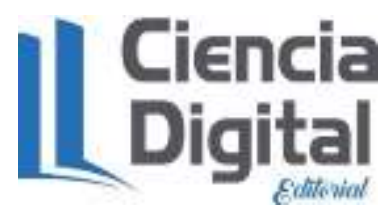


El artículo que se publica es de exclusiva responsabilidad de los autores y no necesariamente reflejan el pensamiento de la Revista Alfa Publicaciones.

\section{Ciencia}

El artículo queda en propiedad de la revista y, por tanto, su publicación parcial y/o total en otro medio tiene que ser autorizado por el director de la Revista Alfa Publicaciones.
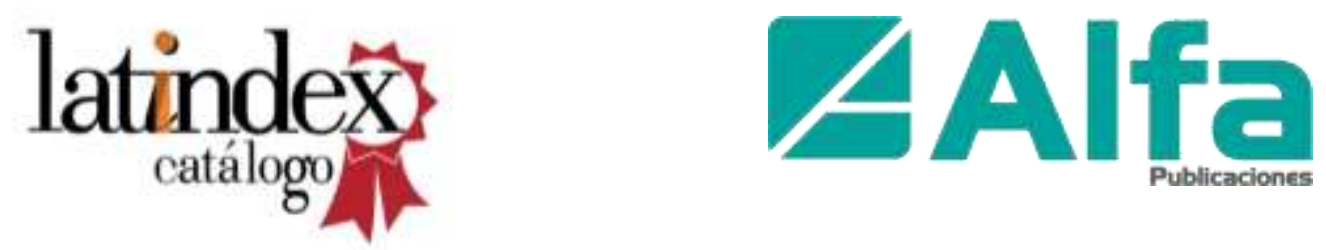

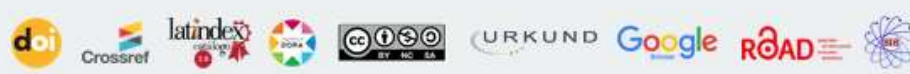
DLatinREV

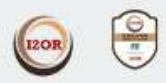

ESJI $=$ REDIB

$\underbrace{}_{\text {wizdom.ai }} \mathrm{O}_{\text {OpenAlRE }}^{+}$

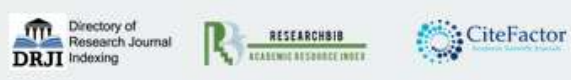

